\title{
PAL 1: ANOTHER YOUNG GLOBULAR CLUSTER?
}

A. ROSENBERG

Osservatorio Astronomico di Padova, Italy.

I. SAVIANE, G. PIOTTO AND S. ZAGGIA

Università di Padova, Dip. di Astronomia, Italy.

AND

A. APARICIO

Instituto de Astrofisica de Canarias, Spain.

\begin{abstract}
We present a color magnitude diagram (CMD) in the $\mathrm{V}$ and I bands reaching $\sim 4$ magnitudes below the turn off $(\mathrm{TO})$ for the galactic globular (?) cluster Pal 1. A comparison with other well-observed clusters and theoretical models suggests that Pal 1 has an age of $8 \pm 2$ Gyrs, which would make it the youngest globular cluster of our Galaxy.
\end{abstract}

The age. $\mathrm{V}$ and I band frames centered on the cluster core, for a total exposure time of $7260 \mathrm{~s}$ and $3630 \mathrm{~s}$ respectively, have been collected at the 2.5m INT (Roque de los Muchachos Observatory, La Palma) for a total field coverage of $11.2 \times 10.3 \mathrm{arcmin}^{2}$. A further field at $10 \mathrm{arcmin}$ from the cluster center has been secured in order to evaluate the background-foreground contamination.

Fig.1 (left) presents the CMD of the internal 1.35 arcmin, where the main features of the diagram are clearly defined. The same figure displays the fiducial sequence for NGC $1851([\mathrm{Fe} / \mathrm{H}]=-1.29$, Age=17 Gyrs, Saviane et al. (1995)), Rup 106 ([Fe/H]=-1.69, Age=12-13 Gyrs, Buonanno et al. (1993), and M67 ([Fe/H]=-0.09, Age=5 Gyrs, Montgomery et al. (1993)). The red giant branch (RGB) falls between those of Rup 106 and M 67. If it's still uncertain metallicity $([\mathrm{Fe} / \mathrm{H}]=-1.01$, as quoted by Webbink (1985)) is close to that of NGC 1851, Pal 1 should be one of the youngest GC's in our Galaxy. In the attempt to put some constraints on the age, we have used Bertelli et al. (1994) models. Due to the impossibility to have an independent estimate of the distance modulus, we have compared the theoretical isochrones with our fiducial points, using the color difference between the 

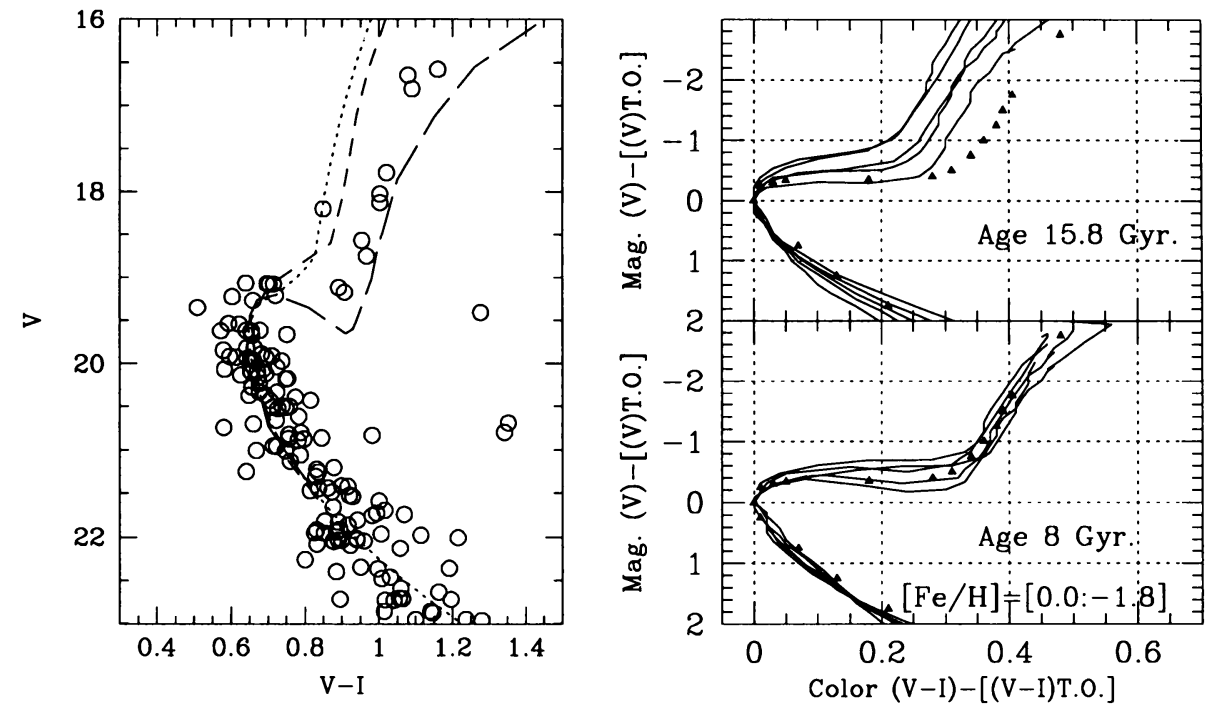

Figure 1.

TO and the SGB $\left(\delta(\mathrm{V}-\mathrm{I})_{\mathrm{TO}}^{\mathrm{SGB}}\right)$. In particular, in this case the difference has been measured fixing the TO position in color and magnitude $\left((\mathrm{V}-\mathrm{I})_{\mathrm{TO}}\right.$ and $\mathrm{V}_{\mathrm{TO}}$ ), and taking a reference point on the SGB $\delta \mathrm{V}$ magnitudes above the $\mathrm{TO},\left((\mathrm{V}-\mathrm{I})_{\mathrm{SGB}}\right.$ and $\left.\mathrm{V}_{\mathrm{SGB}}\right)$, so that $\mathrm{V}_{\mathrm{SGB}}=\mathrm{V}_{\mathrm{TO}}-\delta \mathrm{V}$, and $\delta(\mathrm{V}-\mathrm{I})_{\mathrm{TO}}^{\mathrm{SGB}}$ $=(\mathrm{V}-\mathrm{I})_{\mathrm{SGB}}-(\mathrm{V}-\mathrm{I})_{\mathrm{TO}}$. With different values of $\delta \mathrm{V}(0.8<\delta \mathrm{V}<1.6)$ we find that Pal 1 is consistently very young, precisely, we obtain ages between [6.5-9.5 Gyrs] for $[\mathrm{Fe} / \mathrm{H}]=[0.0:-1.8]$. It is also shown in Fig.1 (right) that a standard $16 \mathrm{Gyr}$ age isochrone cannot match $\mathrm{Pal} 1$ fiducial points for any metallicity in the interval (0.0:-1.8) (upper panel), while a good match with an isochrone of $8 \mathrm{Gyr}$ can be done (lower panel).

The morphological parameters. The density profile of Pal 1 has been obtained from star counts, corrected for completeness and background subtracted. For the first time we have been able to accuratelly correct the counts for the background contamination. Dynamical parameters were obtained by a single-mass isotropic King model fit, giving $c=1.6$ and $r_{c}=14^{\prime \prime}$ making Pal 1 like a normal globular cluster. The central surface brightness is $\mu_{V}=20.85$.

\section{References}

Bertelli G., Bressan A., Chiosi C., Fagotto F. \& Nasi E., 1994, A\&AS, 106, 275.

Buonnano R., Corsi C.E., Pecci F.F., Richer H.B. \& Fahlman G.G., 1993, AJ, 105, 184 Montgomery K.A., Marshall L.A. \& Janes K.A., 1993, AJ, 106, 181

Saviane I., Piotto G., Fagotto F. \& Capacciolli M. (This meeting.)

Webbink R.F., 1985, IAU SYMP 113, 541. 